\title{
Perfectionamiento et démocratisation : regard sur la constitution cubaine après la réforme de 1992
}

Emmanuel Jos

\section{(2) OpenEdition}

Journals

Édition électronique

URL : http://journals.openedition.org/plc/430

DOI : $10.4000 /$ plc. 430

ISSN : 2117-5209

Éditeur

L'Harmattan

Édition imprimée

Date de publication : 1 janvier 1999

Pagination : 9-50

ISSN : 1279-8657

\section{Référence électronique}

Emmanuel Jos, «Perfectionamiento et démocratisation : regard sur la constitution cubaine après la réforme de 1992 », Pouvoirs dans la Caraibe [En ligne], 11 | 1999, mis en ligne le 04 mars 2011, consulté le 30 avril 2019. URL : http://journals.openedition.org/plc/430 ; DOI : 10.4000/plc.430 


\section{Perfectionamiento et démocratisation : regard sur la constitution cubaine après la réforme de 1992}

par Emmanuel JoS

Professeur de droit public Université des Antilles et de la Guyane

Parmi les nombreux écrits consacrés à Cuba, notamment en langue française, il en est très peu qui portent sur les aspects constitutionnels de son régime politique, notamment depuis la révision de 1992. La science économique et la science politique l'emportent largement sur le droit dans les rayons consacrés à Cuba. Pourtant, l'étude de la constitution cubaine offre diverses opportunités. Elle permet d'abord de s'interroger sur la signification de la constitution dans un régime comme celui-là. Elle permet ensuite de mieux appréhender les principes idéologiques et juridiques qui sont mis en avant par les dirigeants du pays et d'apprécier leur degré de spécificité. Elle permet enfin de formuler une hypothèse quant à la dynamique dans laquelle s'inscrit actuellement le régime cubain: transition démocratique ou réaffirmation et approfondissement de la ligne révolutionnaire empruntée depuis 1959 ?

La constitution actuelle de Cuba date de 1976. Elle a été révisée en 1992. Depuis la fin de la dépendance espagnole en 1898, ce pays a connu une succession de régimes politiques et de constitutions qui reflètent une vie politique troublée. Les aspirations à la liberté politique ont été contrariées à maintes reprises par la mise en place de pouvoirs autoritaires, souvent dépendants des Etats Unis d'Amérique. 
Le cycle constitutionnel de l'Etat cubain commence avec la constitution de $1901^{1}$. Bâtie sur le modèle américain, elle est appliquée, tant bien que mal, en dépit de la fraude et de la corruption, jusqu'en 1928, date à laquelle elle subit une réforme permettant la prorogation du mandat de président du général Gérardo Machado, qui ne tarde pas à instituer une dictature rigoureuse. La chute de Machado, le 12 août 1933, inaugure une période troublée et instable caractérisée par une succession d'actes constitutionnels émanant des détenteurs successifs du pouvoir.

Au bénéfice des troubles de l'année 1933, Fulgencio Batista amorce son irrésistible ascension. Il devient chef de l'armée et installe à la présidence le colonel Carlos Mendietta. La constitution de 1901 est abrogée et remplacée par une constitution provisoire le 3 février 1934. Un nouveau texte provisoire est adopté le 11 juin 1935.

Pour faire droit aux aspirations démocratiques de plus en plus fortement exprimées, le 15 novembre 1939, est élue une assemblée constituante. Elle élabore un texte de 286 articles, emprunts de nombreux principes de la démocratie libérale: reconnaissance de droits individuels civils et politiques, mais aussi économiques et sociaux ; contrôle de la constitutionnalité ; recours au référendum ; non continuisme (le Président est élu pour quatre ans et non rééligible avant huit ans) ; séparation des pouvoirs, exécutif bicéphale, bicamérisme, responsabilité ministérielle partielle devant les chambres, sans droit de dissolution. Promulguée le 5 juillet 1940, publiée au journal officiel le 8 juillet, elle entre en vigueur le 10 octobre. Constitution mixte (présidentielle, matinée de parlementarisme) «généreuse et audacieu-

1. Voir: Ramon Infiesta, Historia constitutional de Cuba, La Havane, Editorial Cultural, S.A., 1951, 399 p. Dans cet ouvrage, l'auteur s'intéresse principalement au constitutionnalisme à Cuba pendant la période coloniale, il analyse les courants annexionnistes, réformistes, autonomistes et séparatistes pendant cette période, et termine son parcours par la présentation de la constitution de 1901 puis de l'amendement Platt qui institue un contrôle des Etats Unis sur Cuba. Voir aussi : Andrés Maria Lazcano Y. Mazon, Las constituciones de Cuba, Madrid, Ediciones Cultura Hispanica, 1952; «Corpus constitutionnel», Recueil universel des constitutions en vigueur, Cuba, Tome III, Chapitre 3, Leiden, E. J. Brill, 1983, 67 p. 
$s e$ », selon M. Nicolas Graizeau ${ }^{2}$, consacrant le nationalisme, le droit social, et l'équilibre des pouvoirs, elle se révèle en "pratique inopérante $»^{3}$ et devient davantage le symbole de l'idéal démocratique qu'un véritable instrument de gouvernement ${ }^{4}$.

La période 1940-1952 voit la prééminence du parti authentique de Grau San Martin. Batista interrompt le processus légal en prenant le pouvoir le 10 mars 1952. Sa dictature est renversée par la révolution des «barbudos », conduite par Fidel Castro et Ernesto Guevara, dit le Che, dans la nuit du 31 décembre 1958.

L'un des objectifs de la révolution est de rétablir l'ordre constitutionnel reposant sur la constitution symbole de 1940. Ce ne sera pas tout à fait le cas. La loi fondamentale de la République de Cuba promulguée le 7 février 1959, censée reprendre les dispositions de 1940, apporte en réalité de très profondes modifications, notamment dans le domaine des libertés : suspension du caractère inamovible du pouvoir judiciaire, abandon du principe de non-rétroactivité du droit pénal, suppression de toutes les charges électives, extension de la peine de mort aux délits politiques, suspension des garanties des droits individuels ${ }^{5}$. L'organe principal de l'Etat devient le Conseil des ministres. Il dispose du pouvoir législatif et même constituant. C'est lui qui désigne le Président de la République. Le pouvoir politique se trouve désormais concentré entre les mains des chefs de la révolution, et singulièrement de Fidel Castro. Celui-ci, jusque là ministre de la défense, devient Premier ministre par le décret du 16 février 1959. La présidence devenue formelle (elle ne conserve guère que son droit de veto suspensif) est exercée d'abord par Manuel Urrutia, et après sa démission, pour cause de divergence sur la radicalisation du processus révolutionnaire, par Osvaldo Dorticos Torrado.

2. Nicolas Graizeau, «Genèse, exégèse et pratique de la constitution de 1940 », in Cuba sous le régime de la constitution de 1940, sous la direction de James Cohen et Françoise Moulin Civil, Paris, L'Harmattan, 1997, p. 23-58.

3. Ibidem.

4. Cette dualité entre le symbole démocratique et l'efficacité gouvernementale se vérifie particulièrement s'agissant de la constitution haïtienne de mars 1987.

5. Voir Tisca Jan F., «Constitutions of communist Party States », p. 256 et ss. 
Aidée en cela par les discours et les comportements de plus en plus hostiles du puissant voisin américain, la révolution "vert olive », fortement nationaliste, bascule dans le camp communiste en 1961.

Jusqu'au début des années 70, la construction du socialisme concerne principalement l'économie et l'organisation sociale. Dans le domaine économique, c'est notamment la réforme agraire, la réorganisation du système bancaire, la planification centrale, la réorientation du commerce extérieur. Dans le domaine social, c'est la mise en place des organisations de masse et du parti de la révolution. $\mathrm{Au}$ nombre des organisations de masse on note en particulier la Fédération des femmes cubaines, l'Union des pionniers, l'Association nationale des petits agriculteurs.

Le Parti Uni de la Révolution socialiste naît en février 1963, sous le leadership de Fidel et de Raul Castro.

Le Parti Communiste Cubain (PCC) est créé en octobre 1965. Son Comité central comprend initialement 100 membres. Le Secrétariat en compte 9, et le Bureau Politique 8. Fidel Castro et son frère Raul contrôlent le pouvoir à tous les échelons. Ils sont respectivement premier et deuxième secrétaires.

Le 25 novembre 1972 l'organisation politique est modifiée. Un Comité exécutif du Conseil des ministres de 9 membres, avec à sa tête Fidel Castro, est crée. Chaque membre du Comité dirige un département ministériel. Cette rationalisation permet plus d'efficacité dans la gestion de l'Etat et introduit un élément de collégialité, qui n'enlève rien en pratique à l'autorité personnelle du « lider maximo ».

En 1974 est lancée dans la province de Matanzas une expérience de décentralisation qui servira de base pour l'institutionnalisation de la Révolution. La même année un projet de constitution est mis en chantier sur la base des orientations définies par le Bureau politique et le Comité exécutif du Conseil des ministres. L'année suivante se tient le premier congrès du PCC et le projet de constitution est soumis au Premier ministre. Le texte est publié le 11 avril 1975. Il est discuté par les syndicats et les principales organisations de masse. Il est soumis à l'avis du Congrès, puis au référendum le 15 février 1976. Il est 
approuvé par 97,7 \% des votants. La Constitution est promulguée le 24 février 1976. Elle entre en application tout de suite.

Les sources intellectuelles de cette constitution résident dans le nationalisme et surtout dans la doctrine marxiste-léniniste. Celle-ci est caractérisée notamment par la critique des libertés formelles, consacrées par les régimes de démocratie libérale, et par le rejet du principe de la séparation des pouvoirs auquel est préféré celui de la concentration du pouvoir entre les mains du prolétariat. La constitution stalinienne de 1936 sert de modèle aux constituants cubains de $1976^{6}$. Ce choix traduit clairement le positionnement idéologique de Cuba, la détermination d'une « règle du jeu » théorique (compte tenu de la forte personnalisation du pouvoir, reconnue unanimement, à travers Fidel Castro) pour la vie politique du pays, mais il a aussi, et peut être surtout, une signification internationale. Comme le note M. Jean-Louis Seurin «le modèle stalinien de 1936... sera systématiquement repris par les gouvernements révolutionnaires qui se montraient soucieux de rompre leur isolement international $»^{7}$.

Pendant dix ans (1976-1986) le régime ne connaît pas de changements notables. L'adoption d'une nouvelle constitution en URSS en 1977 n'entraîne pas d'adaptation mimétique à Cuba.

A partir du milieu des années 80, Cuba va de crises en crises et au début des années 90, beaucoup d'écrits pronostiquent la fin de Fidel Castro et de la Révolution cubaine ${ }^{8}$. D’ajustements économiques en ajustements politiques et institutionnels, le régime réussit à se pérenniser, mais demeure fragile.

6. Sur l'évolution du constitutionnalisme soviétique voir : «L'union soviétique », numéro spécial de Pouvoirs, ${ }^{\circ}$ 16, 1978 ; Maurice Lesage, Le système politique de l'URSS, 1987 ; Patrice Gélard, La constitution de l'URSS, 1991 ; Daniel Colas, Les constitutions de l'URSS et de la Russie, 1997.

7. Jean-Louis Seurin, «Des fonctions politiques des constitutions, pour une théorie politique des constitutions » in Le constitutionnalisme aujourd'hui, sous la direction de Jean-Louis Seurin, Paris, 1984, p. 51.

8. Jean-François Fogel, Bertrand Rosenthal, Fin de siècle à la Havane, Seuil, 1993 ; Irving Horowitz et alii, Cuba communism 1959-1995, Transaction Publischers, 1995. 
Une accumulation de facteurs adverses génère, en effet, des crises successives auxquelles le régime doit faire face en amorçant un certain nombre de réformes, plus ou moins profondes ${ }^{9}$. La perestroïka et la glasnost, initiées par Gorbachev en Union soviétique, les contreperformances dues à la bureaucratisation, les comportements de corruption, l'accumulation de difficultés économiques générées par le blocus américain et un contexte économique international défavorable, l'exode des balseros, conduisent les dirigeants à rechercher la voie d'une nouvelle impulsion. Il ne sera toutefois question ni de restructuration ni de transparence, à l'instar du grand frère soviétique, mais de «rectification des erreurs et des tendances négatives ${ }^{10}$.

Dans le domaine politique, il s'agit moins de changer d'orientation que de se remettre dans l'axe de la révolution.

Dans le domaine économique, il ne s'agit pas tout à fait de la mise en place d'un programme d'ajustement structurel selon les recettes du FMI et de la Banque Mondiale, compte tenu de l'inspiration néo-libérale de ceux-ci, mais il s'agit bien d'un programme de redressement fondé sur l'austérité. En 1986 la dette de Cuba vis à vis des pays de l'Ouest s'élève à 6 milliards de dollars et le pays a du la renégocier avec le Club de Paris.

Dans le domaine social, la réaffirmation des valeurs morales traditionnelles constitue une priorité.

Mais l'appareil dirigeant est bientôt lui-même sujet à une crise majeure. Le 12 juin 1989 le général Ochoa est arrêté pour corruption et malversation ainsi que d'autres officiers. Il est accusé d'avoir des liens avec des narcotrafiquants internationaux. Le 7 juillet il est condamné à

9. Voir Ramonet Ignacio, «Cuba : Rénovation de la Révolution ?», Le Monde diplomatique, septembre 1985 , p. 2-4.

10. Voir discours de Fidel Castro, Granma, 11 janvier 1987, pp. 2-5 et $1^{\text {er }}$ février 1987, pp. 2-4. Voir aussi Janette Habel, Rupture à Cuba, le castrisme en crise, Paris, La Brèche, 1989, en particulier le Chapitre III «Crise sociale, bureaucratisation et rectification ». Pour marquer l'originalité du processus cubain, la population a utilisé l'expression de «castroïka » cf. Denis Hautin-Guiraut, «Cuba à l'heure de la "castroïka" », Le Monde, samedi 25 juin 1986, p. 1 et 3. 
mort par un tribunal militaire spécial ainsi que Antonio de la Guardia, Amado Padron et Jorge Martinez. La sentence est confirmée par le Conseil d'Etat, sous la présidence de Fidel Castro. L'exécution est annoncée par Granma le 13 juillet $^{11}$.

Au début des années 90, l'implosion du bloc de l'Est, auquel Cuba était arrimé par des liens divers (accords commerciaux ${ }^{12}$, $\mathrm{CAEM}^{13}$ etc.) qui entraîne la perte dune série d'avantages économiques et stratégiques, le durcissement de l'attitude des Etats Unis à travers notamment la loi Torricelli (renforcée quelques années après par la loi Helms-Burton), les conséquences économiques désastreuses des intempéries, conduisent Fidel Castro et les dirigeants cubains à instaurer une période dite «spéciale » en temps de paix. Par analogie avec les périodes spéciales en temps de guerre, il s'agit de sauver la patrie en danger. Ceci passe par la mobilisation populaire pour faire face à la crise ${ }^{14}$. Des citadins sont envoyés aux champs pour renforcer la main d'œuvre agricole, les transports sont réduits, l'électricité est rationnée, l'usage de la bicyclette s'intensifie, le cheval prend parfois le relais du tracteur. Des changements apparaissent dans les orientations économiques du pays (introduction du dollar, accent mis sur le développement du tourisme, ouverture partielle au capital étranger).

Dans le domaine institutionnel, un certain nombre de modifications constitutionnelles, relayées par une nouvelle loi électorale, sont décidées au cours du quatrième Congrès du Parti. Des discutions sont organisées sur la réforme de la constitution, en 1991. Les modifications sont approuvées par l'Assemblée nationale du

11. Voir la chronologie dans Conflict and change in Cuba, edited by Enrique A. Baloyra and James A. Morris, p. 314.

12. Voir Crosnier Marie-Agnès, «La dépendance économique de Cuba », Le Courrier des Pays de l'Est, $\mathrm{n}^{\circ} 239$, avril 1980, p. 3-40.

13. Voir Perez Silvia, «Cuba en el CAME, una integración extracontinental», Nueva Sociedad, septiembre/octubre, 1983, n 68, p. 131-139.

14. Voir Janette Habel, «Rectification dans la rectification?», Problèmes d'Amérique Latine, $\mathrm{n}^{\circ}$ 99, janvier mars 1991, p. 3-16; Alfred Padula, «Cuban Socialism », in Conflict and change in Cuba, op. cit., p. 31-34. 
pouvoir populaire au cours de la onzième session ordinaire de la troisième législature qui se tient les 10,11 et 12 juillet $1992^{15}$.

Comment appréhender cette réforme institutionnelle ? Traduitelle un changement d'orientation idéologique? Amorce-t-elle une transition démocratique ou confirme-t-elle la ligne révolutionnaire des dirigeants cubains qui entendent promouvoir un autre type de démocratie, différent de celui qui prévaut dans la plupart des grandes puissances occidentales?

L'exégèse du nouveau texte constitutionnel de Cuba, et l'examen de la pratique, notamment en matière de droits de l'homme et d'élections, nous poussent à penser qu'il n'y a pas à Cuba de transition vers le modèle de la démocratie représentative, mais volonté de perfectionnement (perfectionamiento) du régime révolutionnaire ébauché en 1959. Ce parti pris d'organisation particulière du pouvoir s'inscrit délibérément dans une orientation idéologique déclarée, clairement explicitée dans le texte même de la constitution. La réforme de 1992 n'apporte pas sur ces différents points des changements radicaux. Elle reflète la volonté des dirigeants cubains de sauver leur système en l'actualisant. La question de fond demeure donc celle de l'appréciation de la démocratisation du régime cubain.

\section{I. - UNE IDEOLOGIE REVOLUTIONNAIRE DECLAREE : LE «MARTISME-LENINISME »}

Deux idéologies imprègnent fortement, et explicitement, la constitution cubaine: le marxisme-léninisme et le martisme. La première est prépondérante dans le texte de 1976 et le reste après la révision de 1992, mais plus que jamais le culte voué à José Marti apparaît essentiel à côté de la référence à Marx, Engels et Lénine. La formule de M. Olivier Duhamel se justifie aujourd'hui plus encore qu'hier, c'est bien de «martisme-léninisme » qu'il s'agit à Cuba sous

15. Granma, 11 juillet 1992, p. $1-4 ; 13$ juillet 1992, p. $3 ; 8$ novembre 1992, p. 2. 
Fidel Castro ${ }^{16}$. Quelle relation y a-t-il entre ces deux doctrines ? Le lien est celui de la Révolution. Les dirigeants cubains, Fidel Castro le premier, s'estiment les héritiers de ceux qui ont lutté pour l'indépendance de Cuba et se veulent communistes parce que révolutionnaires.

\section{A. - La fidélité réaffirmée au socialisme marxiste-léniniste}

L'article premier de la constitution affirme sans ambiguïté que «Cuba est un Etat socialiste ». Il l'est clairement à travers les principes qui régissent sa vie politique, économique et sociale; il l'est dans la façon de reconnaître et d'organiser le régime des libertés publiques; il l'est dans les orientations essentielles de ses relations internationales. L'abandon du socialisme dans l'Europe de l'Est n'a pas conduit à une suppression de la référence mais à une sorte de dépoussiérage des formulations, traduisant une renonciation à des concepts devenus archaïques. La réforme constitutionnelle de 1992 n'apporte donc que des modifications secondaires au système, mais dans la mesure où les nouvelles formulations prennent de la distance avec la rédaction inspirée par la constitution soviétique de 1936, on peut y voir, toutes proportions gardées, une manière tardive de déstalinisation.

16. Olivier Duhamel, «Castro », in Les grands révolutionnaires, Les feux de l'Amérique latine, Martinsart, 1978, p. 229, Robert Escarpit avait lui aussi souligné l'importance du martisme à Cuba, en écrivant dans Le Monde du 9-10 mai 1976 : «Le cas de José Marti est étrange. La pensée clairvoyante et forte de ce républicain libéral a été intégrée à l'idéologie de la révolution cubaine comme le signe spécifique de son identité. On parle couramment de «marxisme-léninisme-martianisme », et il n'est pas interdit de penser que c'est par le dernier terme que la doctrine s'enracine le plus profondément dans la conscience nationale ». 


\section{1. - Dépoussiérage partiel de la conception de l'Etat}

La réaffirmation du caractère socialiste de l'Etat s'accompagne de l'abandon des formules qui tendaient à en faire un Etat de classe. En effet, là où la version initiale de l'article premier précisait qu'il était l'Etat «des ouvriers et des paysans, unis aux autres travailleurs manuels et intellectuels », la version révisée se contente de dire qu'il est l'Etat «de travailleurs», et ajoute «organisé avec tous pour le bien de tous », formule empruntée à José Marti ${ }^{17}$. L'abandon de la conception de classe apparaît plus nettement encore avec la suppression pure et simple du deuxième paragraphe de l'ancien article 4 qui disait: "le pouvoir du peuple travailleur repose sur l'alliance inébranlable de la classe ouvrière, des paysans et des autres couches laborieuses de la ville et de la campagne, sous la direction de la classe ouvrière ».

L'article 3 nouveau (rédaction 1992) renoue avec le vocabulaire constitutionnel classique. Il n'est plus dit que «tout le pouvoir appartient au peuple travailleur» mais que «la souveraineté réside dans le peuple». La nouvelle formulation se rapproche des formulations classiques que l'on trouve dans la plupart des constitutions occidentales. Faut-il y voir un signe en leur direction, destiné à rendre le régime plus acceptable à leurs yeux ?

Le Parti Communiste Cubain, parti unique, demeure l'avantgarde et la force dirigeante de la société et de $1^{\text {'Etat }}{ }^{18}$. Il ne gère pas l'Etat. Ce n'est pas un parti de masse ${ }^{19}$. Ses membres sont recrutés selon une sélection stricte. C'est lui «qui organise et oriente les efforts communs vers les objectifs élevés de la construction du socialisme et l'avancé vers la société communiste ». Toutefois, il n'est plus présenté,

17. Liceo de Tampa, 26 novembre 1891.

18. On trouvera une version française des Statuts du Parti Communiste de Cuba dans «Corpus Constitutionnel », Recueil universel des constitutions en vigueur, Cuba, Tome III, Leiden E. J. Brill, 1983, p. 55-64.

19. Conformément à la conception léniniste du parti définie notamment dans Que Faire? 
depuis la révision de 1992, comme l'avant-garde de la classe ouvrière $^{20}$, mais comme l'avant-garde de la nation cubaine, comme souligné supra. Là aussi est abandonnée la perspective de classe, devenue trop archaïque.

L'article 6 est, quant à lui, partiellement réécrit afin de gommer l'impression d'embrigadement de la jeunesse par le parti. L'accent est mis désormais sur la formation des jeunes à prendre des responsabilités pour le plus grand bien de la société cubaine.

D'après le texte constitutionnel, l'Etat socialiste cubain continue de reconnaître et d'encourager les organisations de masse et sociales qui ont émergé des luttes passées et qui continuent à jouer un rôle important dans la construction et la défense de la société socialiste.

Pour ce qui a trait aux relations internationales, l'article 11 de la constitution affirmait le rattachement de Cuba «à la communauté socialiste mondiale », autrement dit au bloc communiste. Consécutivement à la chute du communisme dans les pays de l'Est, cet énoncé a été purement et simplement supprimé. Pour la même raison, Cuba ne fait plus sien le principe de «l'internationalisme prolétarien », l'accent est mis désormais sur l'anti-impérialisme et l'internationalisme tout court ${ }^{21}$.

\section{2. - Libéralisation économique à dose homéopathique}

Pour ce qui est du système économique, après la réforme de 1992, on observe quelques modifications liées à l'évolution du contexte.

La constitution prend à son compte la perspective du développement durable et souligne le devoir de l'Etat et des citoyens de protéger l'environnement et les ressources naturelles du pays ${ }^{22}$. Le

20. Ancien article 5.

21. Article 12.

22. Article 27. 
commerce extérieur n'est plus «assumé exclusivement par l'Etat ${ }^{23}$. Celui-ci se contente désormais de le diriger et de le contrôler ${ }^{24}$.

Et surtout, l'article 23 reconnaît désormais la possibilité de propriété privée au sein des «entreprises mixtes » et «des sociétés et associations économiques qui se constituent conformément à la loi ». Une ouverture très nette est faite ainsi en direction des entreprises étrangères désirant investir à Cuba. Cette orientation est confirmée par la loi du 5 septembre 1995 sur les investissements, adoptée par l'Assemblée Nationale du Pouvoir Populaire, après de très nombreux débats. L'ouverture du pays au capital étranger reste, néanmoins, subordonnée à un certain nombre de principes: non atteinte à la souveraineté de l'Etat cubain; non-exploitation des travailleurs cubains ; préservation des ressources naturelles de l'île ; exclusion des secteurs de la santé, de l'éducation, et de la défense ; socialisation des profits individuels ${ }^{25}$.

Mais en dépit de ces modifications, le principe de base du régime cubain reste celui de la propriété socialiste de tout le peuple sur les moyens fondamentaux de production ${ }^{26}$, et la planification centrale demeure $^{27}$.

\section{3. - Relativité des droits civils et politiques et «précarisation » des droits économiques, sociaux et culturels}

Dans le domaine des libertés publiques, l'accent est mis par le régime cubain sur les droits économiques et sociaux. Les droits civils

23. Ancien article 18 .

24. Nouvelle rédaction de l'article 18.

25. Arnold August, Democracy in Cuba and the 1997-98 elections, Canada, Editorial José Marti, 1999, p. 227.

26. Article 14. A noter que les petits agriculteurs ont la propriété de leurs terres (article 19), et que chacun garde ses propriétés d'usage personnel (article 21).

27. Article 16. 
et politiques ne sont pas ignorés. Ces derniers sont, toutefois, consacrés de façon relative et les premiers, après avoir représenté la vitrine de la Révolution, connaissent une certaine «précarisation», du fait de la conjoncture économique de plus en plus défavorable.

Sont théoriquement reconnus par la constitution cubaine la plupart des grandes libertés classiques telles que la liberté de parole, de la presse, de réunion, d'association, et de manifestation. Ces droits restent toutefois subordonnés aux exigences du socialisme. S'agissant de la liberté de la presse, l'article 53 affirme explicitement que sa reconnaissance se fait conformément «aux objectifs de la société socialiste ». Bien plus, au motif d'assurer aux citoyens l'exercice effectif de cette liberté «la presse, la radio, la télévision, le cinéma et les autres mass média appartiennent à l'Etat ou à la société, et ne relèvent en aucun cas de la propriété privée, ce qui garantit leur utilisation au service exclusif du peuple travailleur et dans l'intérêt de la société ».

D'une façon générale, l'article 10 de la constitution affirme l'obligation pour chacun de respecter strictement la légalité socialiste. L'infraction à ce principe est d'ailleurs punissable ${ }^{28}$.

L'application très large de ce principe contribue à rendre la situation des droits de l'homme à Cuba extrêmement préoccupante. Les rapports d'Amnesty International ne cessent de dénoncer les violations graves des libertés politiques et la pratique abusive de la peine de mort à $\mathrm{Cuba}^{29}$. De multiples résolutions des Nations Unies ont exprimé également l'extrême préoccupation de la communauté

28. Article 62.

29. Voir par exemple : Rapport annuel d'Amnesty pour 1998, p.148-151. Voir aussi : Antoine Spire, «Le pouvoir absolu du lider maximo», La chronique d'Amnesty, $\mathrm{n}^{\circ}$ 131, 1997, p. 16-17. Dans cet article, M. Spire qui est journaliste dénonce en particulier l'absence de liberté de la presse à cuba. Il écrit notamment : « pour l'autorité cubaine, les droits à la libre expression se résument à la glorification de la révolution de $1961 »$. Il note que des centaines de personnes sont incarcérées pour délit d'opinion, que les journalistes sont harcelés, emprisonnés ou condamnés à l'exil. Selon lui, La population se clochardise, la prostitution, notamment estudiantine, se généralise, la délinquance et la mafia gagnent du terrain. 
internationale $^{30}$. La Communauté européenne, tout en se montrant favorable au développement des relations avec Cuba, se montre, elle aussi, très désireuse d'un progrès sur le terrain des droits de l'homme ${ }^{31}$.

Certes, on note favorablement l'inclusion, par la révision de 1992, d'un article 8 qui dit que «L'Etat reconnaît, respecte et garantit la liberté religieuse », alors que l'ancien article 54 prévoyait que « La loi réglemente les activités des institutions religieuses » et qu'il était «illégal et punissable d'opposer la foi ou la croyance religieuse à la Révolution ». Il y a un changement d'attitude incontestable du régime vis à vis de la religion, notamment catholique. Le Pape est reçu par Fidel Castro en 1998 et les chrétiens peuvent retrouver leurs pratiques

30. La Commission des Droits de l'Homme des Nations Unies a prorogé le mandat du Rapporteur spécial chargé d'examiner la situation des droits de l'homme à Cuba par sa résolution 1996/69 du 29 avril 1996. Sur la base de son rapport (E/ CN. 4/1997/53) elle a adopté le 16 avril 1997 la résolution $1997 / 62$ par 19 voix contre 10 et 24 abstentions (vote par appel nominal). Dans cette résolution la Commission se déclare préoccupée par la permanence à Cuba de violations des droits de l'homme et des libertés fondamentales énumérés dans la Déclaration universelle des droits de l'homme, tels que les libertés de pensée, de conscience et de religion, d'opinion et d'expression, de réunion et d'association ainsi que les droits associés à l'administration de la justice. La Commission se déclare aussi consternée par la violation du droit à la vie commise par le Gouvernement cubain lorsqu'il a abattu deux aéronefs civils non armés le 24 février 1996. Elle demande au gouvernement cubain d'autoriser les partis politiques et les organisations non gouvernementales à exercer librement leurs activités dans le pays en réformant la législation dans ce domaine; de mettre fin à l'emprisonnement de militants des droits de l'homme, d'ouvrir les prisons aux organisations humanitaires non gouvernementales, et de libérer les nombreuses personnes qui ont été arrêtées pour activités politiques. Elle invite, enfin le gouvernement cubain à veiller à ce que les droits des travailleurs soient garantis, notamment dans le cadre de système de négociation collective indépendants et généralisés.

31. Danielle Perrot, «La Communauté européenne et le développement démocratique en Amérique Latine », Cahiers de l'Administration Outre-mer, n ${ }^{\circ}$ 6/7, 1994-1995, p. 63-77. En décembre 1996, l'Union européenne a adopté une position commune stigmatisant le non respect des droits de l'homme à Cuba. Toutefois, dans le cadre de la négociation du nouvel accord avec les Etats ACP devant faire suite à la convention de Lomé IV, Cuba a obtenu le statut d'observateur, rompant ainsi son isolement, l'UE se réservant la possibilité d'ouvrir avec le gouvernement de ce pays un dialogue politique tendant à améliorer la situation. 
liturgiques traditionnelles. Mais cette hirondelle ne fait pas le printemps des droits de l'homme à Cuba. Il reste que toutes les libertés peuvent être limitées, voire supprimées au nom de l'intérêt du socialisme, et c'est le Parti, et plus précisément son «lider» qui dit ce qui est bon pour le socialisme.

Par ailleurs, l'absence d'autorité judiciaire véritablement indépendante ne permet pas une protection efficace des libertés par les juges. Certes, la constitution cubaine affirme que «les juges sont indépendants dans l'exercice de leurs fonctions et ne doivent obéissance qu'à la loi » ${ }^{32}$, mais jusqu'où va l'indépendance effective de la justice dans ce système, quand on sait que les juges sont élus, et qu'ils peuvent être révoqués par ceux qui les ont élus ${ }^{33}$ ?

S'agissant de la réglementation des libertés en période exceptionnelle, la révision de 1992 esquisse apparemment un rapprochement avec les constitutions occidentales qui prévoient des modalités particulières pour ces périodes. Elle institue, en effet, un régime constitutionnel d'état d'urgence ${ }^{34}$. Toutefois, on observe que cet article ne vise pas à poser des garde-fous vis-à-vis d'un recours abusif à l'état d'urgence, ni à donner des garanties constitutionnelles minimales aux citoyens pendant cette période. Il donne pouvoir au Président du Conseil d'Etat (en l'occurrence Fidel Castro) pour déclarer l'état d'urgence, se contente d'énoncer de façon très large les cas où l'état d'urgence peut être déclaré (catastrophes naturelles et circonstances diverses où l'ordre intérieur, la sécurité du pays ou la stabilité de l'Etat se trouvent affectés), et confie à la loi le soin de préciser les conditions de forme pour la déclaration, les effets, la date d'achèvement, «de même que les droits et devoirs fondamentaux reconnus par la constitution dont l'exercice sera réglé de façon différente pendant la durée d'application de l'état d'urgence ».

Les dirigeants cubains se défendent en mettant en avant les performances en matière de droits sociaux, et justifient la suspension

32. Article 122 .

33. Article 126.

34. Article 67. 
de certaines libertés par la légitime défense contre l'agression qu'ils subissent de la part des Etats Unis d'Amérique sous la forme d'un blocus économique et d'une propagande anti-castriste acharnée ${ }^{35}$.

De fait, une attention particulière est portée par la constitution cubaine sur la famille ${ }^{36}$, la condition féminine ${ }^{37}$, la santé ${ }^{38}$, l'éducation et la culture ${ }^{39}$, et les conditions de travail ${ }^{40}$. Dans la ligne de la critique marxiste du caractère formel des libertés bourgeoises, sont précisés par le texte les voies et moyens par lesquels ces libertés peuvent devenir effectives.

On a pu mettre au crédit du régime cubain la réalisation de performances remarquables et incontestables dans le domaine social et culturel. Les indicateurs en matière d'éducation et de santé ont été parmi les meilleurs au sein des pays en développement. En 1992, l'espérance de vie à la naissance était de 75,7 ans, le taux d'alphabétisation de $95,7 \%$, il y avait un médecin pour 300 habitants ${ }^{41}$ le taux de scolarisation atteignait $97 \%$ pour le primaire, $93 \%$ pour le secondaire, tout cela en dépit d'un produit intérieur brut par habitant assez faible (3 $400 \$$ EU en 1995) ${ }^{42}$. Les effets de la crise économique se font toutefois sentir de plus en plus. Dans ce nouveau contexte, il s'avère que les droits économiques et sociaux qui ont fait la fierté du régime et son attrait vis à vis de l'extérieur connaissent une précarité de plus en plus marquée. Le secteur de la santé est particulièrement

35. «Le message de cuba sur les Droits de l'homme», discours du Vice-président Carlos Lage à Genève devant la Commission des Droits de l'homme des Nations Unies, Granma, 28 mars 1999, p. 10-13. Ce texte stigmatise notamment l'adoption contre Cuba des lois Torricelli et Helms-Burton.

36. Chapitre IV.

37. Article 44.

38. Article 50.

39. Chapitre V.

40. Articles 45 à 47.

41. Les médecins cubains sont les rares spécialistes de certaines maladies telles que le vitiligo.

42. PNUD, Rapport sur le développement humain, 1998, et Maryse Roux, Cuba, Karthala, 1997, p. 5 et 79-82. 
touché. Les médicaments et le matériel médical commencent à manquer cruellement. La population cherche notamment un palliatif en se tournant vers les plantes médicinales ${ }^{43}$.

\section{B. - La valorisation du martisme}

Les analyses de l'évolution du régime cubain pendant la dernière décennie l'ont peu souligné, pourtant il y a là un élément important pour la compréhension du régime : le martisme est devenu à Cuba une idéologie de premier plan. Il convient de s'interroger sur sa signification et sur sa fonction.

1. - Amplification des références à José Marti depuis 1992

L'explicitation de la référence à José Marti dans la constitution cubaine s'affirme plus nettement après la révision de 1992. De façon très significative, alors que la version initiale du texte disait «Guidés par la doctrine victorieuse du marxisme-léninisme », la version révisée de 1992 énonce « Guidés par la conception de José Marti et les idées politico-sociales de Marx, Engels et Lénine ». Pour les auteurs du texte, il est clair qu'après l'implosion du communisme dans les pays de l'Est, il est devenu surréaliste de parler de «victoire du marxismeléninisme ». Mais en dépit de l'échec d'un mouvement historique, les constituants cubains estiment qu'il demeure possible de réaffirmer leur attachement aux idées de Marx, Engels et Lénine. Ce faisant, ils mentionnent également José Marti.

Dans le corps de la constitution on retrouve très clairement la volonté d'un rattachement désormais plus explicite encore à l'esprit de José Marti avec la nouvelle formulation de l'article 5. Le texte initial disposait que «Le parti communiste de Cuba, avant-garde organisée

43. Ces difficultés sont au premier plan des préoccupations de la population lors des réunions avec les délégués de leurs circonscriptions, voir August, op. cit., p. 382401. 
marxiste-léniniste de la classe ouvrière, est la force dirigeante supérieure de la société et de l'Etat... ». Dans la version révisée de 1992, il est dit «Le parti communiste de Cuba, martiste et marxisteléniniste, avant-garde de la nation cubaine, est la force dirigeante supérieure de la société et de l'Etat... ». On observe d'une part que le parti est qualifié de martiste en plus d'être marxiste léniniste, et d'autre part qu'il n'est plus présenté comme avant-garde de la classe ouvrière, ce qui est relégué au rang des archaïsmes, comme on l'a souligné supra, mais comme avant-garde de la nation cubaine, ce qui met clairement l'accent sur le versant nationaliste de l'idéologie du régime.

S'agissant de l'éducation et de la culture, la révision de 1992 fait apparaître que la politique dans ce domaine est fondée non plus sur «la conception scientifique du monde, établie et développée par le marxisme-léninisme » (version initiale de l'article 38) mais sur «les avancées de la science et de la technologie, et la conception marxiste et martiste, la tradition pédagogique progressiste cubaine et universelle ». Ici se confirme à la fois : la renonciation à l'archaïsme du socialisme scientifique au profit d'une prise en compte des avancées du monde moderne, et l'adjonction du qualificatif martiste à celui de marxiste.

\section{2. - Aspects fondamentaux du martisme}

Dans le texte constitutionnel amendé, deux caractéristiques essentielles du martisme se dégagent : le martisme est un humanisme, le martisme est ensuite un nationalisme révolutionnaire ouvert.

Le martisme est un humanisme. Le préambule de la constitution s'achève par la phrase suivante :

«Nous proclamons notre volonté de voir la loi des lois de la République présidée par ce profond désir, enfin réalisé, de José Marti :

"Je veux que la première loi de notre République soit le culte des Cubains à la dignité absolue de l'homme" ». Homme d'idéal, José Marti a lutté pour la liberté politique de son peuple au nom d'une vision universelle, celle de l'émancipation humaine. 
Le martisme est un nationalisme révolutionnaire ouvert. Dans le préambule de la constitution cubaine, la référence à José Marti est tantôt implicite tantôt explicite. Elle est implicite dans le rappel de l'héritage «des patriotes qui, en 1868, entreprirent la guerre d'indépendance contre le colonialisme espagnol, et de ceux qui, dans un dernier effort, en $1895^{44}$, assurèrent la victoire de 1898 ».

Le martisme peut être considéré comme une des versions latinoaméricaines du nationalisme et de l'anticolonialisme, à l'instar du bolivarisme. Leur nationalisme ne se fonde ni sur l'exaltation d'une race ni sur celle d'un peuple en tant qu'essence close sur elle-même, il se veut ouvert et solidaire. Il est l'expression d'un nationalisme ouvert, dans la mesure où la mise en valeur d'un atout de Cuba, à savoir sa tradition pédagogique progressiste, n'est pas présentée comme étant son apanage.

La référence à la conception martiste du nationalisme explique sans doute l'approche ouverte de la citoyenneté que l'on trouve dans la constitution cubaine. Les articles relatifs à cette question consacrent, en effet, très clairement le droit du $\mathrm{sol}^{45}$. On note, par ailleurs, que parmi ceux qui sont considérés comme citoyens cubains de naissance on trouve «les étrangers qui, en raison de mérites exceptionnels prouvés dans les luttes de libération de Cuba, ont été considérés comme citoyens cubains de naissance », et que parmi les citoyens cubains par naturalisation il y a «ceux qui ont participé à la lutte armée contre la tyrannie renversée le ler janvier 1959, et qui peuvent le prouver dans les formes légales établies». Il s'agit là de l'affirmation d'une citoyenneté découlant de la solidarité révolutionnaire.

Le nationalisme martiste se manifeste aussi dans l'affirmation de la pleine souveraineté de l'Etat cubain sur «tout le territoire national » aussi bien terrestre, îles y compris, que maritime et aérien, ainsi que sur son environnement et toutes ses ressources naturelles et vivantes

44. Le 19 mai 1895 José Marti est tué au combat. Sur José Marti, voir, entre autres, Jean Lamore, José Marti et l'Amérique, Paris, L'Harmattan, 2 vol., 1986.

45. Articles 28 à 33. 
contenues dans ces espaces ${ }^{46}$. Il en résulte que sont considérés comme «illégaux ou nuls les traités, pactes ou concessions qui ont été signés dans des conditions d'inégalité ou qui méconnaissent ou limitent sa souveraineté (celle de la République de Cuba) sur une portion quelconque du territoire national $\gg^{47}$.

Le principe d'ouverture du nationalisme se traduit par ailleurs dans le texte constitutionnel cubain par un positionnement antiimpérialiste et internationaliste ${ }^{48}$. On note que le texte de 1992 met davantage l'accent sur l'attachement de Cuba aux grands principes du droit international, tels qu'ils sont énoncés dans la Charte des Nations Unies et d'autres traités internationaux auxquels Cuba est partie.

\section{3. - La fonction de légitimation du martisme}

Dans le contexte difficile des crises qui se succèdent, le régime risque de perdre de plus en plus son soutien populaire. Les dirigeants cubains trouvent dans le martisme un instrument de légitimation renouvelée de leur pouvoir. Il s'agit d'apparaitre comme les héritiers de ceux qui ont lutté pour l'indépendance nationale. La Révolution castriste entend perpétuer et prolonger l'œuvre entreprise par les glorieux aînés. Trahir la révolution castriste, serait, en quelque sorte, trahir l'histoire.

La réaffirmation de la référence au marxisme-léninisme procède à l'évidence d'une volonté personnelle de Fidel Castro et des dirigeants qui lui sont proches. En revanche la valorisation du martisme rejoint sans aucun doute l'imaginaire populaire et est susceptible de renforcer les soutiens symboliques du régime.

46. Il s'agit là d'une revendication chère aux pays du tiers monde cf. Dominique Rosenberg, Le principe de souveraineté des Etats sur leurs ressources naturelles, Paris, LGDJ, 1983, 395 p.

47. Article 11.

48. Article 12. 


\section{II. - CONTINUITE ET CHANGEMENTS \\ DANS LES INSTITUTIONS POLITIQUES CUBAINES}

La fidélité des dirigeants cubains aux principes du marxismeléninisme a pour conséquence leur attachement indéfectible au mode d'organisation du pouvoir qui a vu le jour dans les pays communistes, et singulièrement en Union Soviétique. La révision de 1992 n'a pas apporté sur ce point des changements profonds. En revanche elle a innové en matière de dévolution du pouvoir. Faut-il y voir l'amorce d'une transition vers le modèle des démocraties occidentales ? A certains égards les innovations rapprochent le système cubain de celui des démocraties occidentales, mais la portée essentielle de ces innovations demeure le renforcement d'un système cubain spécifique de dévolution du pouvoir. Il nous semble donc plus exact de parler de perfectionnement du système que de transition.

\section{A. - Le maintien d'une gestion unifiée du pouvoir politique}

Le pouvoir d'Etat à Cuba comporte deux échelons : un échelon central et un échelon local qui se subdivise à son tour en provinces et municipalités. Les réformes n'ont pratiquement pas touché à l'organisation du pouvoir central, en dépit de certains changements de vocabulaire. $\mathrm{Au}$ second échelon quelques modifications sont intervenues.

\section{1. - Statu quo au sommet}

Le texte constitutionnel de 1976 fait de l'unité du pouvoir et du centralisme démocratique deux principes essentiels de la démocratie socialiste $^{49}$. La réforme de 1992 supprime la référence explicite à ces principes. Certes, ces expressions disparaissent mais l'organisation et

49. Article 66. 
le fonctionnement du pouvoir à Cuba restent inscrits dans la perspective de l'unification et de la centralisation du pouvoir. Il ne s'agit donc que d'une modernisation du langage. En effet la réforme n'a pas instauré la séparation des pouvoirs, et les éléments constitutifs du centralisme démocratique sont maintenus, à savoir: le caractère obligatoire des dispositions adoptées par les organes supérieurs de l'Etat pour les organes inférieurs; la nécessité pour les organes inférieurs de rendre compte de leurs actes et de leur gestion aux organes supérieurs ; la liberté de discussion, l'exercice de la critique et de l'autocritique et la subordination de la minorité à la majorité ${ }^{50}$.

En l'absence d'une organisation fondée sur la séparation des pouvoirs, la logique organique prévaut largement sur la logique fonctionnelle.

L'organe suprême, chargé d'exprimer la volonté souveraine du peuple cubain, est l'Assemblée Nationale du Pouvoir Populaire (ANPP). Dans la pratique cette suprématie s'exerce très largement par son Conseil d'Etat (CE). Celui-ci comprend un Président, qui fait fonction de chef de l'Etat, un Premier Vice-président, cinq Viceprésidents, un Secrétaire et vingt trois autres membres. Un Conseil des ministres assure à Cuba la fonction gouvernementale, au sens d'organe exécutif et administratif ${ }^{51}$. Son Président est le chef du Gouvernement. Il comporte, par ailleurs, un Premier Vice-président, plusieurs Viceprésidents, les ministres, un secrétaire et d'autres membres que détermine la loi. Un Comité exécutif du Conseil des ministres comprend son Président, son Premier Vice-président, les Viceprésidents et les membres supplémentaires que le Président aura désignés. Le Comité exécutif peut exercer les compétences du Conseil des ministres entre ses sessions ${ }^{52}$.

Le dispositif est complété par l'existence d'un Tribunal populaire suprême, d'un Procureur Général et d'un Vice-procureur Général de la République.

50. Article 68.

51. Article 95.

52. Article 97. 
Le pouvoir constituant appartient à l'ANPP, et à elle seule ${ }^{53}$. Les révisions sont adoptées par elle au vote nominal et à la majorité des deux tiers au moins de ses membres. Le référendum n'est prévu que dans trois cas : si la réforme est totale; si elle porte sur la composition et les pouvoirs de l'ANPP ou de son Conseil d'Etat; et si elle concerne les droits et les devoirs consacrés par la constitution ${ }^{54}$.

Le pouvoir législatif revient aussi en propre à l'ANPP ${ }^{55}$. Les autres organes ne le partagent pas mais peuvent y concourir par leurs initiatives : le Conseil d'Etat, le Conseil des ministres, les commissions de l'Assemblée, le Comité nationale de la Centrale des Travailleurs de Cuba, les directions nationales des organisations de masse, le Tribunal populaire suprême (en matière de justice), le Procureur général de la République (pour les questions relevant de sa compétence), et les citoyens eux-mêmes, pour autant qu'ils parviennent à être au moins dix mille pour présenter cette initiative ${ }^{56}$.

Les lois sont votées par les députés à la majorité simple. Il est toujours possible de les soumettre, si l'Assemblée le juge opportun, à l'adoption populaire. Il faut toutefois souligner ici que s'il est vrai qu'en droit l'acte déterminant du pouvoir législatif est le vote de la loi, en pratique l'influence majeure sur le contenu des textes revient à ceux qui les élaborent en amont. Les textes de loi sont généralement aujourd'hui le fruit d'une mise en forme technique par l'administration de la volonté politique du chef du gouvernement. Ceci est particulièrement vrai à Cuba, les lois y sont l'expression de la volonté politique du lider maximo.

Les lois entrent en vigueur à la date indiquée dans leur texte. Elles sont publiées au journal officiel de la République de Cuba.

En cas de nécessité, c'est le Conseil d'Etat qui est compétent pour donner une interprétation générale et obligatoire des lois en vigueur.

53. Article 70.

54. Article 137.

55. Article 70.

56. Article 88. 
S'apparentent aux lois, mais avec rang inférieur dans la hiérarchie des normes, les décrets-lois. Il n'y a pas dans la constitution de répartition des matières entre lois et décrets-lois. Les décrets-lois se définissent donc comme les règles adoptées par le Conseil d'Etat dans l'intervalle des sessions de l'ANPP. Cette dernière peut révoquer les décrets-lois en totalité ou en partie, au moment de l'une de ses sessions $^{57}$. Compte tenu de l'uniformité idéologique qui prévaut à Cuba, l'occurrence d'un rejet des décrets-lois est hautement improbable. Ce dispositif est favorable à la transformation de l'Assemblée en chambre d'enregistrement ${ }^{58}$.

Le pouvoir de décision budgétaire appartient à l'ANPP, le projet de budget étant élaboré par le Conseil des ministres.

Appartient à l'Assemblée, également, le pouvoir de décision en matière de planification du développement, à partir de projets élaborés par le Conseil des ministres.

Le Conseil d'Etat est chargé d'exécuter certaines décisions de l'Assemblée. Il prend, par exemple, les mesures nécessaires à l'organisation des référendums décidés par elle ${ }^{59}$.

Le pouvoir exécutif-administratif est confié au Conseil des ministres. Il est le gouvernement de $\mathrm{Cuba}^{60}$. C'est lui qui assure la gestion de l'Etat au quotidien. Il prend toutes les mesures nécessaires pour la mise en application des textes normatifs : lois, décrets-lois, budget, plans de développement et autres. Il adopte pour cela les décrets ordinaires. Il dispose de l'administration. Celle-ci est structurée en départements, placés sous l'autorité des ministres.

Dans la conduite des affaires extérieures, l'ANPP n'a que des attributions restreintes. Elle approuve les lignes générales de la

57. L'Assemblée nationale du pouvoir populaire tient deux sessions par an. Leur durée minimale n'est pas précisée. Elles sont convoquées par son Président. La tenue de sessions extraordinaires peut être décidée par le Conseil d'Etat.

58. Corpus Constitutionnel, op. cit., p. 24.

59. Article 90 e.

60. Article 95. 
politique extérieure ${ }^{61}$. Elle décrète l'état de guerre en cas d'agression militaire, et approuve les traités de paix ${ }^{62}$. Son président a compétence pour organiser ses relations internationales ${ }^{63}$. Dans le domaine des relations extérieures les attributions essentielles se répartissent entre le Conseil d'Etat, son Président, et le Conseil des ministres. L'analyse de cette répartition met en lumière, plus encore que dans les autres domaines, le rôle prépondérant du Chef de l'Etat, Président du Conseil d'Etat, Chef du gouvernement, Commandant suprême des Forces armées révolutionnaires. En effet, outre le fait que les attributions collégiales respectives du Conseil d'Etat et du Conseil des ministres s'exercent sous son autorité, il dispose de pouvoirs propres.

C'est lui qui organise et dirige les activités du Conseil d'Etat ainsi que celles du Conseil des ministres. Avec le Conseil d'Etat, il décrète la mobilisation générale, déclare la guerre, signe la paix en substitution à l'Assemblée si nécessaire, désigne les représentants diplomatiques de Cuba, les démet de leurs fonctions, ratifie les traités, les dénonce. Avec le Conseil des ministres, il dirige la politique extérieure et les relations avec les autres gouvernements, approuve les traités avant de les soumettre à ratification, dirige et contrôle le commerce extérieur, pourvoit à la défense nationale, accorde l'asile territorial. Au titre de ses attributions propres, il reçoit les lettres de créances des chefs des missions diplomatiques étrangères, et assume le commandement suprême des Forces armées révolutionnaires.

Le pouvoir judiciaire n'est pas l'apanage des tribunaux. L'ANPP dispose en effet du pouvoir de juger en matière de constitutionnalité des lois, des décrets-lois, des décrets, et des autres dispositions générales $^{64}$. En matière de loi, elle est donc clairement juge et partie.

Les juges jouissent d'une indépendance fonctionnelle, mais restent sous l'emprise du pouvoir politique qui les nomme et peut les

61. Article $75 \mathrm{~h}$.

62. Article $75 \mathrm{i}$.

63. Article $81 \mathrm{~d}$.

64. Article 75 c. 
révoquer. De plus le Conseil d'Etat a la faculté de donner des instructions au Tribunal populaire suprême.

Le Ministère public a en charge le contrôle de la légalité. Après la révision de 1992 il n'est plus précisé socialiste, ce qui laisse entrevoir une volonté de détacher quelque peu la justice de l'idéologie. Il doit veiller au respect par tous de la constitution (ceci a été ajouté en 1992), des lois et des autres dispositions juridiques. Il est, toutefois, subordonné à l'ANPP, mais à elle seule. Le Procureur général, quant à lui, doit rendre compte de sa gestion à l'ANPP, et reçoit des instructions directes du Conseil d'Etat.

\section{2. - Innovations partielles à la base}

A l'échelon local, le pouvoir est réparti entre les Assemblées Provinciales du Pouvoir Populaire (APPP) et les Assemblées Municipales du Pouvoir Populaire (AMPP). Elles participent à l'exercice du pouvoir d'Etat ${ }^{65}$. Le schéma institutionnel cubain est donc sur ce point différent de celui de la décentralisation telle qu'elle est instituée dans un pays comme la France, où les collectivités territoriales n'exercent pas de pouvoir d'Etat. Depuis 1992, ont été mis en place des Conseils populaires et des Conseils de défense provinciaux et municipaux, ainsi que des zones de défense. Les Conseils populaires sont constitués dans les villes, les villages, les quartiers et les zones rurales. Ils regroupent les délégués élus dans les circonscriptions et peuvent comprendre les représentants des organisations de masse et des institutions les plus importantes des secteurs concernés ${ }^{66}$. Leur fonction est de faire participer la population à la recherche des solutions aux problèmes qu'elles rencontrent, de dynamiser les actions de développement, et faciliter la coordination entre les acteurs. Celle des Conseils de défense est de préparer, en temps de paix, le pays à faire face aux situations de guerre, par une mobilisation générale, et en s'inscrivant dans le plan général de

65. Article 103.

66. Article 104. 
défense. Ces deux structures nouvelles s'expliquent par l'acuité des difficultés économiques rencontrées par Cuba depuis la perte de ses soutiens financiers et commerciaux du bloc de l'Est, et par la crainte renforcée d'une agression extérieure.

Les APPP, au nombre de 14, ont pour tâche notamment de faire appliquer les lois et autres dispositions adoptées par les organes centraux, de mettre en œuvre la partie du plan qui les concerne, d'adopter le budget de leur province, de contrôler l'administration provinciale (et les différents organismes qui s'y rattachent) dans les secteurs de l'éducation, de la santé, de la culture, du sport, de l'environnement, et de fortifier la légalité, l'ordre ainsi que la capacité de défense du pays.

Les AMPP, au nombre de 169, ont des attributions semblables aux APPP, mais circonscrites à leurs propres territoires. A la différence de ce qui se passe dans la plupart des pays, à Cuba les municipalités ne correspondent pas aux villes. En vertu de l'article 102 de la constitution, nouvellement rédigé, le découpage tient compte des nécessités en matière de relations économiques et sociales de la population et de la capacité à satisfaire les besoins locaux minimum ${ }^{67}$.

Les APPP et les AMPP disposent de Comités Exécutifs qui représentent ces assemblées pendant les inter-sessions. Ces organes permanents fixent l'ordre du jour des assemblées et organisent leurs travaux. Ils exercent un contrôle sur les activités sociales et économiques dans leur zone. Ils sont en relation directe avec les services administratifs de l'Etat sur place. Ceux-ci sont soumis à une double subordination : aux organes centraux et aux organes locaux du pouvoir d'Etat.

Pour contrebalancer les effets de la bureaucratisation, des Commissions de travail composées de délégués élus et de simples citoyens sont organisées. Elles réunissent des informations et rédigent des rapports sur la qualité des services sociaux ou sur les activités

67. Ainsi, la capitale La Havane ne constitue pas un ensemble municipal, et n'a pas de maire comme beaucoup de grandes villes dans le monde. La cité de La Havane constitue en revanche une province. 
économiques territoriales. Elles peuvent s'auto-saisir ou agir sur demande des assemblées ou du Comité exécutif. Leurs recommandations peuvent être reprises par les instances de décision et devenir des obligations.

Pour favoriser la participation populaire au pouvoir ${ }^{68}$, des réunions de compte-rendu se tiennent tous les six mois dans les circonscriptions électorales. Elles sont présidées par le délégué de la circonscription. Celui-ci indique les actions qu'il mène. Un débat peut s'engager sur son compte-rendu. Les participants (généralement 50 à $60 \%$ des électeurs de la circonscription) peuvent aussi présenter des requêtes. Les échanges portent essentiellement sur les problèmes rencontrés dans la vie quotidienne.

$\mathrm{Au}$ total, le maintien du système de gestion du pouvoir établi depuis 1976 indique qu'il n'y a pas de transition dans ce domaine à Cuba. Les modifications intervenues dans les modalités de dévolution du pouvoir autorisent-elles, en revanche, à parler de transition ?

\section{B. - L'évolution du système électoral : transition démocratique ou perfectionamiento?}

Avant 1992, les électeurs désignaient les représentants aux assemblées politiques selon un système de paliers successifs. Etaient élus d'abord les membres des Assemblées municipales. Ceux-ci désignaient à leur tour les délégués aux Assemblées provinciales du pouvoir populaire d'une part, et à l'Assemblée nationale du pouvoir populaire d'autre part. Les juges étaient choisis à chaque échelon par

68. Sur la participation populaire au pouvoir à l'échelon municipal à Cuba voir les travaux du Centre d'Etudes pour les Amériques, en particulier l'article de Haroldo Dilla, Gerardo Gonzalez Nunez, Anna Teresa Vicentelli, «Participación y desarollo en los municipios cubanos », in Cuba en crisis, Jorge Rodriguez Beruff (compilador), Editorial de la universidad de Puerto Rico, 1995, p. 59-100. 
les assemblées politiques ${ }^{69}$. Des innovations importantes ont été introduites par la révision de 1992 et par la loi électorale $n^{\circ} 72$ du 29 octobre 1992. Comment qualifier ce réformisme? Certains y voient l'amorce d'une transition démocratique, autrement dit d'un rapprochement du système cubain avec les pratiques électorales des démocraties occidentales. Pour les dirigeants cubains, il n'en est rien. Il s'agit du perfectionamiento d'un système qui se veut spécifique et qu'ils considèrent comme une des manifestations les plus réussies de la transformation révolutionnaire de Cuba. Il s'agit donc d'un réformisme de consolidation, non de transition.

L'étude du système électoral cubain permet de confirmer son originalité marquée. Toutefois, son appréciation débouche, selon nous, sur des conclusions contrastées.

\section{1. - Une spécificité marquée}

La spécificité du système apparaît surtout dans le choix des candidats et à un degré moindre dans l'élection proprement dite.

Les membres des 169 Assemblées municipales du pouvoir populaire sont élus pour deux ans et demi. Les membres des 14 Assemblées provinciales du pouvoir populaire et les 601 députés de l'ANPP sont élus pour cinq ans.

A certains termes les trois assemblées sont renouvelées, il y a donc élections générales, à d'autres, seules les assemblées municipales le sont. On parle alors d'élections intermédiaires. Les dernières élections étaient des élections générales. Le processus de renouvellement des trois assemblées s'est déroulé sur neuf mois, entre le 13 juin 1997 et le 24 février $1998^{70}$.

69. Sur le système tel qu'il résulte de la constitution de 1976, voir André et Francine Demichel, Cuba, dans la série Comment ils sont gouvernés, Paris, L.G.D.J., 1979 , p. $135-205$ et $287-333$.

70. Voir annexe. 
Le processus commence par les élections aux Assemblées municipales du pouvoir populaire. Elles sont convoquées par le Conseil d'Etat. Est mise en place aussitôt une Commission Electorale Nationale. Celle-ci veille à l'organisation des élections. Elle est présidée par le ministre de la justice et comprend par ailleurs un viceprésident et quatorze membres. Ils sont nommés par le Conseil d'Etat, choisis parmi les personnalités publiques. Selon un système de concertation entre la base et le sommet des Commissions Electorales locales sont mises en place aux différents échelons. La subdivision va jusqu' aux circonscriptions. Elles étaient au nombre de 14533 en 1997. Les Comités de la Révolution jouent un rôle important dans la proposition des membres de ces commissions. Le bénévolat est la règle. Les commissions de circonscription ont la charge d'effectuer les inscriptions sur les listes électorales. Le corps électoral cubain est composé des nationaux âgés de plus de seize ans. On note que la majorité électorale à Cuba est plus basse que dans la plupart des pays.

Vient ensuite, l'une des phases les plus originales du système cubain à savoir le choix des candidats. Officiellement les candidatures aux assemblées municipales sont régies par les principes suivants: indépendance par rapport au parti et aux associations de masse, droit égal de tous les citoyens à être élus, droit de proposition ouvert à tous ceux qui figurent sur la même liste électorale. Pour mettre en œuvre ces principes, chaque circonscription est divisée en petites zones de nomination (au maximum 8) par les soins des Commissions électorales locales, sous le contrôle de la Commission électorale nationale. Sur convocation et sous la présidence de la Commission électorale de circonscription se tiennent, dans les lieux les plus aptes à rassembler les citoyens concernés, des assemblées publiques de nomination. Les citoyens y font leurs propositions et les justifient. Par vote à main levée le candidat de la zone est retenu. On peut être candidat dans plusieurs zones. Cette présélection par zones doit déboucher sur la désignation d'au moins deux candidats par circonscription. En pratique dans $88,7 \%$ des circonscriptions se dégagent deux candidats ${ }^{71}$.

71. Arnold August, op. cit., p. 270. 
Une information officielle, notamment sous forme d'affichage des curriculum vitae des candidats, tient lieu de campagne électorale.

Pour l'élection proprement dite le mode de scrutin s'avère assez classique, il s'agit d'un scrutin majoritaire à deux tours. Le vainqueur est celui qui réalise au premier tour plus de $50 \%$ des voix. Au deuxième tour, qui a lieu une semaine après, et auquel seuls les deux candidats arrivés en tête peuvent se présenter, la majorité relative suffit. La participation est généralement forte : $97,59 \%$ en $1997^{72}$. En pratique les candidats sont élus dans la plupart des circonscriptions dès le premier tour. En 1995 il a fallu un deuxième tour dans 332 circonscriptions seulement. En 1997, ce nombre a sensiblement augmenté. Il est passé à 1098 sur les 14533 circonscriptions existantes $^{73}$.

Avant la réforme de 1992, l'élection des délégués aux Assemblées Municipales du Pouvoir Populaire revêtait d'autant plus d'importance que ceux-ci avaient le privilège d'élire les membres des Assemblées provinciales et de l'Assemblée nationale. Désormais, et c'est la réforme essentielle, les élus de ces assemblées sont choisis directement par les électeurs au scrutin secret. Toutefois, il demeure que $50 \%$ des membres de l'Assemblée nationale du pouvoir populaire doivent être des élus municipaux. Ceci conditionne une partie des candidatures.

Vu l'enjeu idéologique et politique plus important de l'élection des députés, le choix des candidats est plus encadré que pour les municipales. En effet, les candidatures sont proposées par les plénums des organisations de masse. Seuls les citoyens âgés d'au moins 18 ans sont éligibles. Les propositions sont recueillies par la Commission Nationale des Candidatures qui opère la sélection. Avant 1992, cette commission était présidée par un membre du PCC. Désormais, c'est la Centrale des Travailleurs cubains qui désigne son président.

Les membres des 14 Assemblées provinciales, et les 601 députés à l'Assemblée nationale sont élus le même jour selon le même mode de

72. Ibidem, p. 295.

73. Ibidem. 
scrutin que pour les municipales. La participation est là aussi très forte et le nombre de bulletins blancs ou nuls demeure minime. En 1998 il a été recensé 94,98\% de suffrages exprimés, 3,36\% de bulletins blancs et $1,66 \%$ de votes nuls ${ }^{74}$.

Dès sa mise en place, l'ANPP élit son Président, son Viceprésident et son Secrétaire, puis sont choisis en son sein les députés qui doivent siéger au Conseil d'Etat pour cinq ans. Celui-ci comprend, il convient de le rappeler, un Président, qui fait fonction de chef de l'Etat et de chef du Gouvernement, un Premier Vice-président, cinq Viceprésidents, un Secrétaire et vingt trois autres membres.

A l'issue des élections législatives du 24 février 1998, les 595 députés présents ont réélu Ricardo Alarcon Président de l'Assemblée et reconduit, sans surprise, à l'unanimité, Fidel Castro à la tête du Conseil d'Etat et son frère Raul Castro comme Premier Vice-président. Le reste du Conseil d'Etat a connu un renouvellement d'environ $45 \%$ de ses membres (14 nouveaux membres sur 31$)^{75}$.

S'agissant du Conseil des ministres, qui assume, on l'a vu, la fonction gouvernementale, au sens d'organe exécutif et administratif, son Président est le chef de l'Etat (en l'occurrence Fidel Castro). Ses autres membres sont désignés par l'ANPP, sur proposition du Président du CE.

Le Président, les Vice-présidents et les autres membres du Tribunal populaire suprême sont aussi élus par l'ANPP, tout comme le Procureur Général et le Vice-Procureur Général de la République.

\section{2. - Ombres et lumières $d u$ perfectionamiento}

Le régime politique cubain est sans aucun doute l'un des régimes actuels les plus controversés. L'une des raisons de la difficulté

74. Ibidem, p. 360-361.

75. Voir Granma, 8 mars 1998, p. 3. 
à porter sur lui un regard neutre est qu'il s'agit d'un régime à très forte intensité idéologique, mais aussi affective.

Il y a dix ans, M. François Maspero notait déjà que les nombreux écrits sur Cuba allaient «des condamnations sans appel au nom de la démocratie et des droits de l'homme, jusqu'aux adhésions admiratives au nom du droit le plus élémentaire de l'homme à ne pas crever de faim, en passant par toutes les nuances de l'indulgence et des jugements balancés » ${ }^{76}$.

Les condamnations sans appel viennent, bien entendu principalement des Etats Unis d'Amérique. Ce pays livre, depuis de nombreuses années, une guerre sans merci au régime politique cubain. Dans le rapport de 1997 du Département d'Etat sur les droits de l'homme à Cuba on peut lire :

«Cuba est un Etat totalitaire contrôlé par le Président Fidel Castro, qui est chef de l'Etat, chef du gouvernement, Premier Secrétaire du Parti Communiste, et commandant en chef des forces armées. Le Président Castro exerce son contrôle sur tous les aspects de la vie cubaine, à travers le Parti Communiste et les organisations de masse qui lui sont affiliées, la bureaucratie gouvernementale, et l'appareil de sécurité d'Etat. Le Parti communiste est la seule entité politique légale, et le Président Castro approuve personnellement l'appartenance au Bureau Politique, groupe sélectionné qui dirige le Parti. Le Parti contrôle tous les postes gouvernementaux, y compris les fonctions judiciaires $\gg 77$.

On note également que les lois dites Torricelli (1992) et HelmsBurton (1996) se veulent des lois sur le rétablissement de la démocratie à Cuba. La dernière s'intitule très précisément «Cuban Liberty and Democratic Solidarity (LIBERTAD) Act $»^{78}$. L'article 201 de cette loi

76. Préface de l'ouvrage de Janette Habel, Ruptures à Cuba, op. cit., p. 25.

77. Cité par August, op. cit., p. 234.

78. Texte publié par les Documents d'actualité Internationale, $\mathrm{n}^{\circ} 16$ du 15 août 1996, p. 674-689. Sur ce texte voir Lowenfeld A., Agora, « The libertad Act. Congress and Cuba : the Helms-Burton Act », AJIL, 1996, p. 418 et s ; Glacett B., « Title III of the Helms-Burton Act is consistent with international law », AJIL, 1996, p. 434 et s. ; Jean-Marc Sorel, «Remarques sur l'application, extraterritoriale du droit national à la 
énonce les «mesures politiques en vue d'un gouvernement de transition et d'un gouvernement démocratiquement élu à Cuba ». Plus éclairant encore, l'article 206 précise les «conditions requises pour déterminer l'existence d'un gouvernement démocratiquement élu». Selon les auteurs de la loi, il convient de mettre en place à Cuba un gouvernement issu d'élections libres et équitables (sous le contrôle d'observateurs internationalement reconnus, dans un processus où les partis d'opposition disposent des moyens de faire campagne, avec un plein accès aux médias), qui respecte les droits fondamentaux, et s'oriente vers un système économique obéissant aux lois du marché et fondé sur le droit de posséder des biens et d'en tirer les fruits. Ce gouvernement devra procéder aux réformes constitutionnelles nécessaires, mettre en place un système judiciaire indépendant et restituer aux Américains leurs biens ou leur verser un dédommagement équitable. Les intérêts américains ne sont donc pas oubliés dans l'énoncé des critères du gouvernement démocratique.

Pour l'essentiel la position américaine consiste à dénier tout caractère démocratique au régime cubain, et les réformes du début des années 90 ne sauraient être vues comme des avancées dans le bon sens. Le point de vue des dirigeants cubains est bien évidemment très différent.

L'orientation démocratique de la Révolution cubaine était affirmée au hasard d'une phrase dans la version de la constitution datant de 1976. Le préambule énonçait, en effet, que la Révolution, dirigée par Fidel Castro, avait «réalisé les transformations démocratiques ». Dans le nouveau contexte du début des années 90, les dirigeants cubains apparaissent soucieux d'affirmer très nettement le caractère démocratique de leur régime. Ainsi, la révision de 1992 offre une nouvelle rédaction de l'article 1. Là où il était écrit « $L a$ République de Cuba est un Etat socialiste d'ouvriers et de paysans, unis aux autres travailleurs manuels et intellectuels », on lit désormais «Cuba est un Etat socialiste de travailleurs, indépendant et souverain,

lumière de la législation américaine récente », Revue Juridique de l'Ouest, 1996, 18 p. ; Brigitte Stern, «Vers la mondialisation juridique ? Les lois Helms-Burton et d'Amato-Kennedy », RGDIP, 1996/4, p. 979-1003. 
organisé avec tous et pour le bien de tous, comme république unitaire et démocratique, pour la production de la liberté politique, la justice sociale, le bien être individuel et collectif et la solidarité humaine».

A l'époque de la Révolution triomphante, les dirigeants cubains étaient portés à considérer leur régime comme un modèle à exporter, un idéal à imiter. Au début des années 90, ils réclament plus humblement le droit à la spécificité. Selon M. Arnold August, les Cubains ne présentent pas leur démocratie et leur processus politique comme un modèle pour les autres. Il cite à cet effet la déclaration de M. Ricardo Alarcon, président de l'ANPP, devant l'Assemblée générale des Nations Unies le 5 novembre 1997: «Nous ne voulons pas être vus comme un modèle. Nous respectons le droit des autres à développer leur propre système, tout comme nous demandons avec force que le nôtre soit respecté ${ }^{79}$.

La réflexion des autorités cubaines sur la démocratie comporte deux aspects. Il s'agit selon eux d'éviter les contradictions du système représentatif et multipartisan tel qu'il a fonctionné à Cuba avant la Révolution : clientélisme, contrôle extérieur, corruption, inefficacité économique. Et il convient en revanche de favoriser une démocratie unitaire et participative.

Unitaire, car tous les citoyens doivent être mobilisés dans un même but. La société est perçue comme un tout homogène. Le multipartisme apparaît comme une source de division nuisible à la construction d'un ensemble solidaire. La démocratie n'apparaît pas ici comme un système de compromis entre les intérêts antagonistes présents dans le corps social ${ }^{80}$. L'exaltation de l'unité s'exprime dans cette déclaration de Fidel Castro rapportée par Mme Janette Habel: «L'idéal c'est l'unité d'opinion, l'unité de doctrine, l'unité des forces, et l'unité de commandement, comme dans une guerre ${ }^{81}$.

79. August, op. cit., p. 250.

80. Sur cette conception de la démocratie voir Thierry Michalon, «A la recherche de la légitimité de l'Etat », RFDC, 34, 1998, p. 289-313.

81. Janette Habel, Ruptures à Cuba, op. cit., p. 129. 
Participative, car les citoyens sont associés étroitement à la dévolution et à l'exercice du pouvoir. Les autorités cubaines considèrent leur système électoral comme l'une des meilleures illustrations de ce type de démocratie.

Pour les dirigeants cubains, les réformes récentes s'inscrivent dans la ligne du projet de la Révolution. Loin de traduire un changement d'orientation, elles sont dans la continuité des transformations amorcées dans les années 60 et prolongées dans les années 70 .

Au-delà de ce clivage extrême - lecture américaine contre lecture cubaine - comment apprécier le système cubain sans faire œuvre partisane ? Quel critère lui appliquer ? A quoi faut-il le comparer? A la situation d'avant la Révolution ? A d'autres pays en développement? Aux pays occidentaux développés ?

Du point de vue de l'histoire des Caraïbes, les ombres et les lumières de la Révolution cubaine ont été très tôt appréciées par les leaders politiques de la région. Parmi ces appréciations, on peut rappeler, en particulier, celle d'Eric Williams, dans l'un de ses principaux ouvrages de référence, à savoir De Christophe Colomb à Fidel Castro : L'histoire des Caraibes (1492-1969). L'auteur ne cache pas son admiration pour certains aspects du nouveau régime mais dénonce son orientation totalitaire. «Cuba-écrit-il - premier pays des Caraïbes à lancer avec succès un défi à la puissance des Etats-Unis dans l'hémisphère, chercha à établir un régime fondé sur l'indépendance nationale et la justice sociale sans oublier l'égalité raciale... l'envers de la médaille est que Cuba est aussi le premier pays des Carä̈bes à introduire un régime totalitaire dans la région, même si le Nouveau Monde peut sembler n'être qu'une collection de dictatures brutales et néfastes ${ }^{82}$. Pour Eric Williams le régime cubain ne saurait être classé, au moment où il écrit, parmi les régimes démocratiques. Son jugement serait probablement peu différent aujourd'hui.

82. Eric Williams, De Christophe Colomb à Fidel Castro: L'histoire des Caraibes, (1492-1969), Paris, Présence Africaine, 1975, p. 535-536. L'auteur consacre, par ailleurs, un chapitre au Castrisme, p. 505-524. 
Parmi les questions de fond, il y a, en effet, celles de savoir à quelle conception de la démocratie on se réfère, et en quoi consiste un processus de démocratisation. La doctrine est abondante sur ce point ${ }^{83}$, et il convient de se situer.

On retiendra, avec M. Guy Hermet, que la démocratie comporte à la fois un aspect procédural et un aspect substantiel. Elle réside écritil dans «la possibilité effective pour les gouvernés de choisir et de renverser leurs dirigeants par le truchement d'élections suffisamment compétitives, tenues à intervalles réguliers et obligatoires, par surcroît non entachées de fraude massive ou de violence contre certains candidats tout autant que contre les électeurs. Elle suppose simultanément le développement de normes juridiques qui protègent les citoyens contre l'arbitraire du pouvoir... Elle requiert, enfin, la mise en cuvre de politiques ou, plutôt, d'un projet global, visant à appuyer le progrès civique, culturel et matériel de la population... » ${ }^{84}$. On admettra volontiers aussi avec lui qu' "elle apparaît également comme une tâche toujours inachevée, non seulement dans les pays qui s'y convertissent aujourd'hui mais, aussi, dans ceux qui estiment, à tort évidemment, l'avoir édifiée de longue date ${ }^{85}$.

Pour le même auteur, «la démocratisation désigne l'abandon d'un mode de gouvernement autoritaire au profit d'un régime libéral, reposant sur le consentement des gouvernés ${ }^{86}$. C'est l'aspect procédural. Il convient donc d'ajouter qu'un processus de démocratisation sur le plan substantiel rejoint finalement le

83. Voir par exemple: Olivier Duhamel, Les démocraties, régime, histoire, exigences, Seuil, 1993; Dominique Gaxie, La démocratie représentative, Clefs, Montchrestien, 1993 ; Alain Touraine, Qu'est ce que la démocratie ?, Fayard, 1994.

84. Guy Hermet, Culture et démocratie, UNESCO, Albin Michel, 1993, p. 31-32.

85. Ibidem. Les regards critiques sur les démocraties libérales occidentales ne manquent pas, voir entre autres un regard de l'intérieur: Alain Minc, L'ivresse démocratique, Gallimard, 1995. Ne manquent pas non plus les critiques portant sur l'occidentalisation du monde par l'implantation systématique et généralisée partout du modèle occidental de la démocratie représentative. Voir dans ce sens: Sous la direction de Sophia Mappa, Développer par la démocratie ? Injonction occidentale et exigences planétaires, Karthala, 1995.

86. Guy Hermet, Le passage à la démocratie, Presse de la Fondation Nationale de Sciences Politiques, 1996, p. 25. 
développement dans la mesure où il inclut l'amélioration des conditions de vie de la population ${ }^{87}$.

Il résulte de ces positionnements qu'aucun régime n'est exempt de reproches, mais il en est sans doute qui en méritent plus que d'autres.

Nous retiendrons qu'il convient sans conteste de déplorer l'excessive personnalisation du régime cubain à travers Fidel Castro. Le non-respect de certaines libertés fondamentales apparait des plus inacceptables ${ }^{88}$. Le contrôle indirect du Parti sur la dévolution du pouvoir, à travers les organisations de masse, en particulier les Comités de la Révolution, nous semble aussi évident. Et l'instauration de l'élection des députés au suffrage universel direct, et au scrutin secret, ne revêt pas la portée démocratique que l'on serait tenté spontanément de lui conférer, compte tenu du fait que cette réforme s'inscrit dans un cadre non pluraliste.

En revanche, il nous semble que le régime cubain peut retenir positivement l'attention, en tant que lieu d'expérimentation de modalités originales de participation populaire. Le processus mis en place pour le choix des candidats et l'élection des délégués aux Assemblées Municipales, par exemple, mérite davantage qu'un rejet en bloc, pour raison de leurre démocratique. Une équipe de chercheurs de l'université de Porto Rico l'a bien compris en y consacrant une étude

87. Emmanuel Jos, « Démocratisation et développement dans la Caraïbe », CAOM, $\mathrm{n}^{\circ} 4,1991$, p. 7-32.

88. Après quelques mesures en faveur des prisonniers d'opinion adoptées en 1998 (voir le Rapport d'Amnesty International de 1999), depuis le début de l'année 1999, la situation des dissidents et des journalistes apparaît des plus préoccupante et traduit un durcissement du régime. Ce durcissement s'explique sans doute par sa fragilisation. Il provoque des réactions de plus en plus nombreuses de la part des milieux intéressés. Ainsi le 17 juin 1999, vingt-cinq organes de presse européens, considérant qu'il s'agissait d'une atteinte intolérable à la liberté d'opinion et d'expression, ont demandé la libération de trois journalistes emprisonnés à Cuba pour outrage envers le président Fidel Castro et comportement socialement dangereux. 
fondée sur une enquête de terrain. Il en ressort des conclusions contrastées $^{89}$.

Haroldo Dilla, Gerardo Gonzalez Nunez et Anna Teresa Vicentelli constatent d'abord que ce processus se déroule dans un climat de liberté. Ils reconnaissent que beaucoup d'élus sont en pratique des membres du PCC, mais que le choix des électeurs n'est pas conditionné en premier lieu par des considérations idéologiques. Les Cubains se déterminent avant tout à partir de critères éthiques et relationnels. Les qualités humaines des candidats à la candidature ou des candidats, priment sur leur appartenance ou pas au Parti. En revanche, ce système qui entend éviter la démagogie, la mercantilisation du suffrage, la division de la population, le clientélisme, ne s'avère pas très efficace pour faire émerger des leaders dynamiques et capables d'affronter les défis du pouvoir. Le système, en interdisant de véritables campagnes électorales n'est pas propre à faire émerger des hommes nouveaux, il favorise ceux qui sont déjà connus dans le système. Il débouche par ailleurs sur une sous-représentation des femmes. Mais, il faut le reconnaître, la difficulté à renouveler les élites et la sous-représentation féminine n'est pas propre au système cubain.

$\mathrm{Au}$ début des années 90, la plupart des observateurs pronostiquaient la chute de Fidel Castro et la fin de la Révolution cubaine. Dix ans après, Fidel Castro et son régime sont encore en place, même si ils apparaissent fragilisés. La longévité du régime s'explique, nous semble-t-il, par deux facteurs principaux, l'un externe, l'autre interne.

Le facteur externe est l'attitude délibérément hostile de la puissance américaine, qui loin d'engendrer la perte de soutiens au régime, à tendance à souder chaque fois davantage les Cubains autour de Fidel Castro. Les lois Torricelli et Helms-Burton ont été paradoxalement des atouts pour le régime qui n'a pas eu de mal à stigmatiser devant l'opinion publique interne et mondiale, l'agression

89. Haroldo Dilla, Gerardo Gonzalez Nunez, Anna Teresa Vicentelli, op. cit., p. 73-81. 
délibérée, et internationalement illicite, de la super puissance mondiale contre un petit pays des Caraïbes.

Le facteur interne est celui de la nature particulière du régime. Tous les observateurs reconnaissent le rôle central joué par Fidel Castro. Les uns parlent d'autoritarisme, de communisme bureaucratique, de caudillisme, de dictature militaire, voire de totalitarisme $^{90}$. Les autres identifient l'existence d'un régime original fondé essentiellement sur le charisme de Fidel Castro, et préfèrent parler de «castrisme». L'originalité du régime cubain nous semble effectivement avérée, mais, selon nous, plus encore que de « castrisme », à Cuba, c'est de « fidélisme » qu'il s'agit. Nous sommes en présence d'un régime sans doute à forte intensité idéologique, mais, nous l'avons souligné, à très forte intensité affective.

Le «fidélisme» repose sur le modèle relationnel du frère ${ }^{91}$. L'utilisation du prénom permet d'établir une relation de proximité affective de caractère familial, d'où découle un attachement rendant difficile une éventuelle rupture du lien. Parmi les soutiens du fidélisme, il y a incontestablement, outre celui généré par la propagande organisée $^{92}$, une part d'adhésion populaire librement consentie. Celle-

90. Sur certaines de ces qualifications, voir Alain Blérald, «Les lectures critiques du castrisme : autoritarisme et bureaucratie », CAOM, 1993, p. 48-57.

91. Une comparaison stimulante nous semble intéressante à faire entre le fidélisme et le gaullisme. Le gaullisme est fondé sur le modèle du père et le fidélisme sur le modèle du frère. Par ailleurs, on peut observer que De Gaulle et Fidel Castro ont cultivé leur image de libérateur, de chef, y compris militaire, et ont voulu asseoir leur légitimité sur l'histoire en s'identifiant aux héros fondateurs de l'identité nationale. Tous les deux ont montré une hostilité au régime des partis, mais là où Castro a mis fin à leur libre constitution, De Gaulle a cherché à construire un système susceptible de transcender les effets pervers des partis, «mal inhérent au gouvernement libre » (selon Tocqueville, cité par Claude Emeri, dans Démocratie, autorité, stabilité, in De Gaulle en son siècle, (1. Dans la mémoire des hommes et des peuples), publié par l'Institut Charles De Gaulle, Paris, Plon et La Documentation Française, 1991, p. 204. La comparaison est d'ailleurs esquissée par Fidel Castro lui-même dans une interview au sujet de De Gaulle, reproduite dans l'ouvrage cité De Gaulle en son siècle, p. 50-57.

92. Le fidélisme puise assez largement sa force dans l'utilisation du verbe. Il s'agit en quelque sorte d'une "logocratie". Janette Habel parle de "gouvernement de la parole» (Rupture à cuba, op. cit., p. 129). Les discours de Fidel, reproduits et 
ci vient du fait que Fidel Castro s'est identifié, et a identifié fortement la Révolution, à ce qui est considéré par les Cubains comme le meilleur de leur histoire, à savoir la lutte pour l'émancipation de Cuba par rapport à toute forme de domination externe. Il a fondé sa légitimité sur l'histoire. Fidel Castro a assimilé son œuvre à celle de José Marti et des autres héros nationaux. Il s'est efforcé d'incarner aux yeux du peuple cubain le rejet du lourd passif des régimes corrompus d'avant 1959. Le «fidélisme » tend donc à faire corps avec l'identité cubaine.

Dans ce contexte, la constitution cubaine représente, on l'a vu, un outil idéologique important, tout autant qu'un moyen de fixer la règle $d u$ jeu en matière $d$ 'accession et d'exercice du pouvoir ${ }^{93}$. Cette constitution et la législation électorale qui la complète n'ont pas échappé au courant de réforme qui a traversé Cuba. Mais, il s'est agi, nous l'avons souligné, d'un réformisme de consolidation et non de transition. Quoique fragilisé, le « fidélisme » se trouve réaffirmé.

massivement diffusés par les médias officiels, durent généralement plusieurs heures. Un véritable culte est organisé autour de la personne de Fidel.

93. Pour reprendre la terminologie bien connue de Max Weber (Le savant et le politique, tr. 1959, éd. 10/18, p. 102-103), on peut dire que la constitution apporte un complément « légal-rationnel » au pouvoir «charismatique » de Fidel Castro, le culte des héros du passé et la volonté de s'inscrire dans leur sillage apportant, dans une certaine mesure, la dimension «traditionnelle». 


\section{Résumé}

La constitution cubaine de 1976 et la loi électorale qui la prolonge ont été modifiés dans le contexte des crises successives qu'a connu le régime de Fidel Castro au début des années 90. Ces modifications ont eu pour objet essentiel tout d'abord de mettre l'accent dans le domaine idéologique sur le martisme à côté du marxisme-léninisme, luimême réaffirmé ; ensuite de donner davantage de pouvoirs aux citoyens de base, en leur permettant notamment de désigner désormais les députés à l'Assemblée Nationale du Pouvoir Populaire au suffrage directe et par vote secret. S'agissait-il de l'amorce d'une transition vers la démocratie libérale? Assurément pas. Il s'est agit en réalité de perfectionamiento, c'est à dire d'approfondissement de la ligne révolutionnaire suivie par Cuba depuis 1959. La nature du régime cubain a donc peu évolué et continue de susciter controverses et appréciations très diverses.

\section{Mots-clés}

Castrisme, participation populaire - Constitution, démocratisation - Cuba Droits de l'homme - Elections Marxisme-léninisme, martisme Perfectionamiento - $\quad$ Pluralisme, réformisme constitutionnel - Pouvoir local - Rectification - Socialisme, fidélisme Unité
Abstract

The 1976 Cuban Constitution and the subsequent election act were amended within the context of the successive crises experienced by the Fidel Castro regime in the early 1990s. These amendments aimed essentially at focusing on the ideology of Marti-ism alongside the newly reaffirmed Marxism/Leninism; also at increasing power for basic citizens, in particular, enabling them henceforth to designate elected representatives to the Popular Power National Assembly by direct and secret vote. Was this the beginning of a transition toward liberal democracy? Certainly not. In reality, it was the process of perfectionamiento, that is, a deepening of the revolutionary orientation followed by Cuba since 1959. The nature of the Cuban regime was, therefore, able to develop and continues to incite much controversy and very diversified evaluations. 